\title{
Measurement of Reactive Hydroxyl Radical Species Inside the Biosolutions During Non-thermal Atmospheric Pressure Plasma Jet Bombardment onto the Solution
}

\author{
Yong Hee Kim • Young June Hong • Ku Youn Baik • Gi Chung Kwon • \\ Jin Joo Choi • Guang Sup Cho • Han Sup Uhm • Do Young Kim• \\ Eun Ha Choi
}

Received: 8 September 2013/ Accepted: 26 February 2014/Published online: 13 March 2014

(C) The Author(s) 2014. This article is published with open access at Springerlink.com

\begin{abstract}
Non-thermal atmospheric pressure plasma jet could generate various kinds of radicals on biosolution surfaces as well as inside the biosolutions. The electron temperature and ion density for this non-thermal plasma jet have been measured to be about $0.8 \sim 1.0 \mathrm{eV}$ and $1 \times 10^{13} \mathrm{~cm}^{-3}$ in this experiment, respectively, by atmospheric pressure collisional radiative model and ion collector current. In this context, the hydroxyl $\mathrm{OH}$ radical density inside the biosolutions has been also investigated experimentally by ultraviolet absorption spectroscopy when the Ar non-thermal plasma jet has been bombarded onto them. It is found that the emission and absorption profiles for the other reactive oxygen species such as $\mathrm{NO}(226 \mathrm{~nm})$ and $\mathrm{O}_{2}{ }^{*-}(245 \mathrm{~nm})$ are shown to be very small inside the biosolution in comparison with those for the $\mathrm{OH}$ radical species. It is found that the densities of $\mathrm{OH}$ radical species inside the biosolutions are higher than those on the surface in this experiment. The densities of the $\mathrm{OH}$ radical species inside the deionized water, Dulbecco's modified eagle medium, and phosphate buffered saline are measured to be about $2.1 \times 10^{16}, 1.1 \times 10^{16}$, and $1.0 \times 10^{16} \mathrm{~cm}^{-3}$, respectively, at $2 \mathrm{~mm}$ downstream from the surface under optimized Ar gas flow of $200 \mathrm{sccm}$. It is also found that the critical hydroxyl $\mathrm{OH}$ radical density for the lung cancer $\mathrm{H} 460$ cells to experience an apoptosis is observed to be around $0.3 \times 10^{16} \mathrm{~cm}^{-3}$ under 1 min plasma exposure in this experiment.
\end{abstract}

Keywords Non-thermal atmospheric pressure plasma jet - Atmospheric pressure collisional radiative model · Hydroxyl $\mathrm{OH}$ radical density inside the biosolution - Ultraviolet absorption spectroscopy

\section{Introduction}

For elimination of the limitations for vacuum plasma operation with least amount of energy and cost, so-called non-thermal atmospheric pressure plasma jet (hereafter non-thermal

Y. H. Kim · Y. J. Hong · K. Y. Baik · G. C. Kwon · J. J. Choi · G. S. Cho ·

H. S. Uhm · D. Y. Kim · E. H. Choi $(\bowtie)$

Plasma Bioscience Research Center, Kwangwoon University, Seoul 139-701, South Korea

e-mail: ehchoi@kw.ac.kr 
plasma) has been emerged recently. Also, this kind of non-thermal plasma has been attracted to areas of biotechnology with their diversity of forms for easy use. Especially, a lot of various reactive chemical species could be generated by non-thermal plasma jet. Non-thermal plasmas jets with diverse types have recently been developed and used in emerging novel biomedical applications. Besides its sterilization effects, the first practical studies on plasma-based treatment of chronic wounds or tumor treatment with non-thermal plasma jet has been very promising [1-4]. There are many plasma jets using various electrode configurations. The direct plasma jets with other types of electrodes are widely used for biomedical [5-9], in which the plasma jets with a needle electrode are one of them. For achievements of the technical requirements for medical instrumentation, advanced non-thermal plasma sources must have some electron temperature and requires specified radical species.

Chemically reactive oxygen species (ROS) and reactive nitrogen species (RNS) play an important role in biomedical field during non-thermal plasma treatment. These effects of ROS and RNS on the biological cells are not studied well yet. Based on these situations, the convergence technology of plasma and biology is highly developed and its research range is widely expanded. Of these species, hydroxyl radical $\mathrm{OH}$, hydrogen peroxide $\mathrm{H}_{2} \mathrm{O}_{2}$ as well as nitric oxide NO species could be generated by non-thermal plasma jet, which is operating at the atmospheric pressure when it contacts with the biosolution surface. Moreover, the non-thermal plasma interaction with biosolutions has received increasing attention for their biomedical applications. It is very important to measure the hydroxyl radical $\mathrm{OH}$ density by using the ultraviolet absorption spectroscopy on the biosolutions [10] as well as inside them induced by the non-thermal plasma jet.

Nowadays, the convergent studies of the non-thermal plasma and biomedicines have been actively increased with high research extension and speed. The ROS radicals generated inside the biosolutions are not sufficiently investigated during non-thermal plasma interactions with bio-solutions. Also the basic mechanisms for the ROS generation inside the biosolution are not well known yet during non-thermal plasma bombardment onto the biosolution surface. Therefore, in this paper, we investigate the hydroxyl $\mathrm{OH}$ radical density inside the biosolutions during the non-thermal plasma bombardment on the biosolution surface by ultraviolet absorption spectroscopy as well as electron temperature and density characteristics for argon non-thermal plasma jet by atmospheric pressure collisional radiative spectroscopy [11].

\section{Experimental Procedure}

Figure 1 shows the schematic experimental setup for the needle-typed non-thermal plasma jet operating at the atmospheric pressure, which is bombarding onto the biosolution surface. We have used an argon gas flow in this experiment for the generation of non-thermal plasma jet. Our non-thermal plasma jet consists of needle-type powered electrode whose diameter is $1 \mathrm{~mm}$, which is located by $1 \mathrm{~mm}$ upward from the end of the cylindrical glass tube, whose diameter is $5 \mathrm{~mm}$ in diameter. Grounded electrode is located by $13 \mathrm{~mm}$ away from the end of glass tube tip, which is the rear bottom surface of quartz, in which the water is filled. The distance between the powered electrode and water surface is set by $3 \mathrm{~mm}$. For Ar gas flow rates ranged from 100 to $350 \mathrm{sccm}$, the electron temperature and plasma ion density of non-thermal plasma jet have also been measured by optical emission spectroscopy based on the atmospheric pressure collisional radiative (CR) model [12] and by the electrical ion current signals at the grounded collector electrode passing through the 


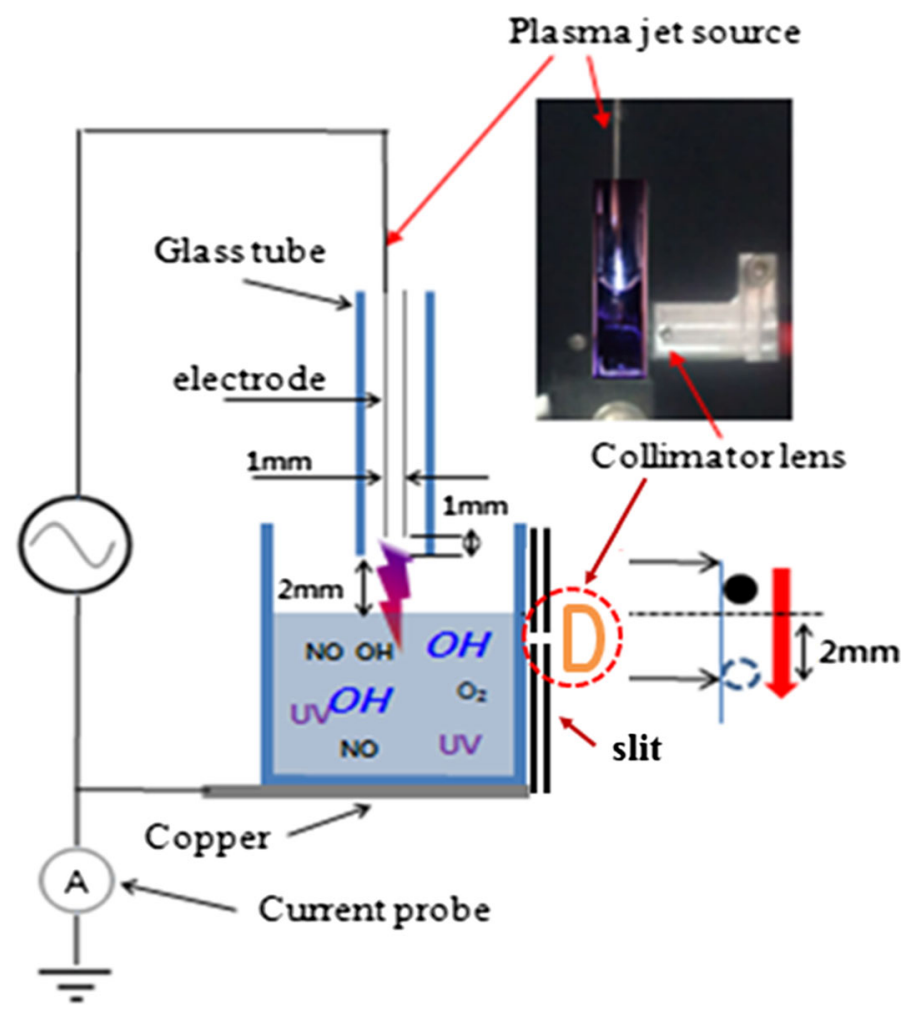

Fig. 1 Schematic experimental setup for the plasma generation for the needle-typed non-thermal argon plasma jet operating at the atmospheric pressure

floating mesh during the non-thermal plasma jet bombardment onto biosolutions. We have investigated the optical emission spectrum emitted from four $2 \mathrm{p}$ metastable Ar emission lines of $696.5,706.7,922.4$, and $912.3 \mathrm{~nm}$ for the measurement of electron temperature based on the atmospheric pressure CR model [11]. The two electrodes in a non-thermal plasma jet have been connected to a square-pulse power supply. Figure 2 shows the voltage and current waveforms applied to the non-thermal plasma jet. It is shown that the voltage signal, $v$, has a root-mean-square voltage of about $1 \mathrm{kV}$ and peak current, $I$, of $34 \mathrm{~mA}$, with current duration of about $2.1 \mu \mathrm{s}$, where the electrical power is given by about $4.9 \mathrm{~W}$, which is obtained from $\frac{1}{\mathrm{~T}} \int_{0}^{\mathrm{T}} i v d \mathrm{t}$, where $\mathrm{T}$ is period of squared pulse with the repetition rate of about $35 \mathrm{kHz}$.

For measurement of hydroxyl $\mathrm{OH}$ radical density, the non-thermal plasma jet has been in contact with the biosolution surface and its optical emission signal has been observed by charge-coupled device (CCD) spectrometer connected by the double slit and optical fiber with collimator lens. Also we have measured the reactive oxygen species, especially, for the hydroxyl $\mathrm{OH}$ radicals by the optical emission spectroscopy as well as its absolute density inside the biosolutions by the ultraviolet (UV) absorption spectroscopy when the non-thermal plasma has been bombarded onto the biosolution surface. Figure 3 shows the schematic experimental setup for the ultraviolet absorption spectroscopy inside the biosolutions. This system consists of deuterium UV lamp in this experiment, whose power is $30 \mathrm{~W}$ with spectral wavelength between 160 and $800 \mathrm{~nm}$, plano-convex lens whose 


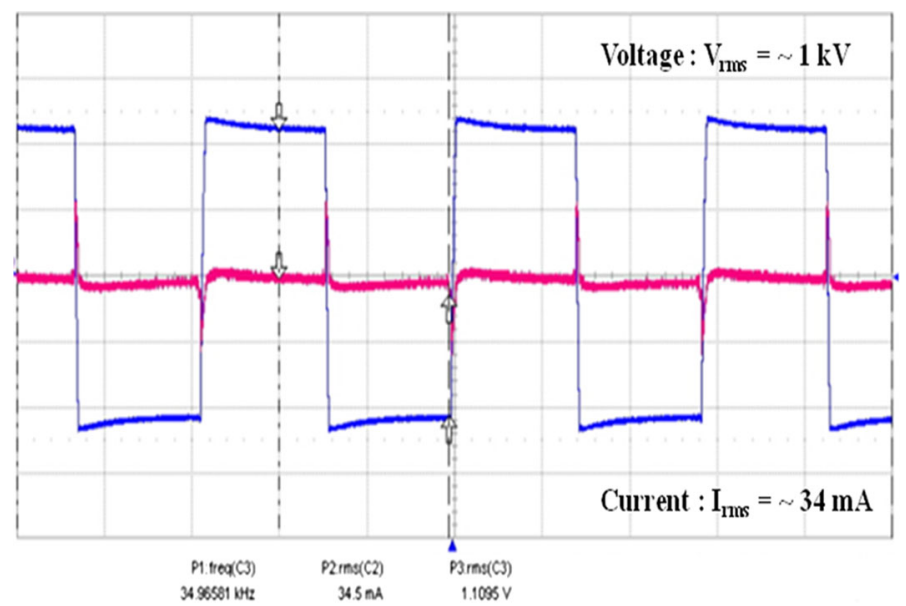

Fig. 2 Electrical voltage and current signals applied to the non-thermal Ar plasma jet

transmission wavelength ranges are from ultraviolet to infrared, double slit connected to the collimator lens, and CCD spectrometer with optical fiber whose spectral range is from 200 to $1,100 \mathrm{~nm}$. The light of UV lamp has been incident and focused by $3 \mathrm{~mm}$ planoconvex lens in front of detector system for the measurement of absorption profiles occurred at $309 \mathrm{~nm}$, of the hydroxyl radical $\mathrm{OH}$ species [4, 12], as shown in the Fig. 3. This detector system consists of double slit in this experiment, whose slit width is $100 \mu \mathrm{m}$ and their gap is $1 \mathrm{~mm}$, and then collimator lens have been connected to the optical fiber whose diameter is $500 \mu \mathrm{m}$. With this experimental situation, we observed no stray light from above the surface since the plasma jet has been bombarded onto the central surface region in the measurement.

The hydroxyl $\mathrm{OH}$ radical density inside the biosolution, which is generated by the argon non-thermal plasma jet interaction with DI water surface, could be obtained by ultraviolet absorption spectroscopy using the Lambert-Beer's law [4]. The incident UV light on the biosolution has the intensity $I_{o}$ and the transmitted light intensity is denoted by $I_{v}$ after passing through the $\mathrm{OH}$ existing region inside quartz tube $(x=10 \mathrm{~mm})$, in which the hydroxyl $\mathrm{OH}$ radical species are generated by the non-thermal plasma bombardment onto the solution surface. The density of hydroxyl radical species, $\mathrm{OH}$, inside the biosolution, which is induced by the non-thermal plasma bombardment onto the biosolution is given by [4]

$$
N=-\frac{1}{\sigma \cdot x} \ln \left(\frac{I_{v}}{I_{0}}\right)
$$

where $N$ is the density or concentration for absorbing species of hydroxyl $\mathrm{OH}$ radicals, $\sigma$ is the cross sectional area of about $1.2 \times 10^{-16} \mathrm{~cm}^{2}$ for absorbing species of hydroxyl radical $\mathrm{OH}$ species [4]. The hydroxyl radical $\mathrm{OH}$ density inside the biosolution could be obtained in Eq. (1), by measurement of the ratio $I_{v} / I_{o}$ of the transmitted intensity to the incident one during non-thermal plasma irradiation onto the biosolution. Here the optimized gas flow condition has been also investigated for maximizing the density of hydroxyl $\mathrm{OH}$ radical 

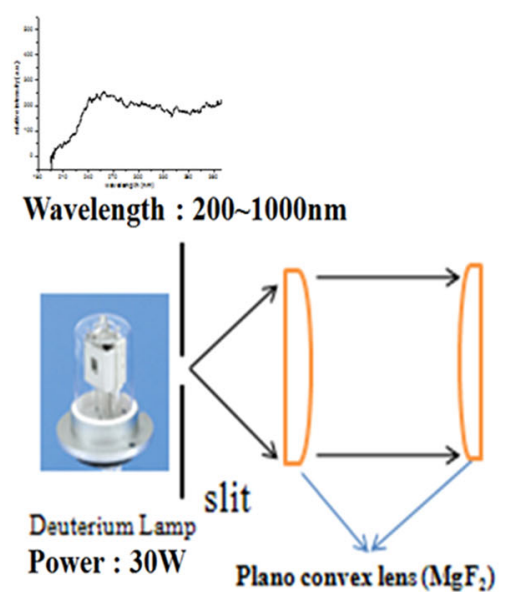

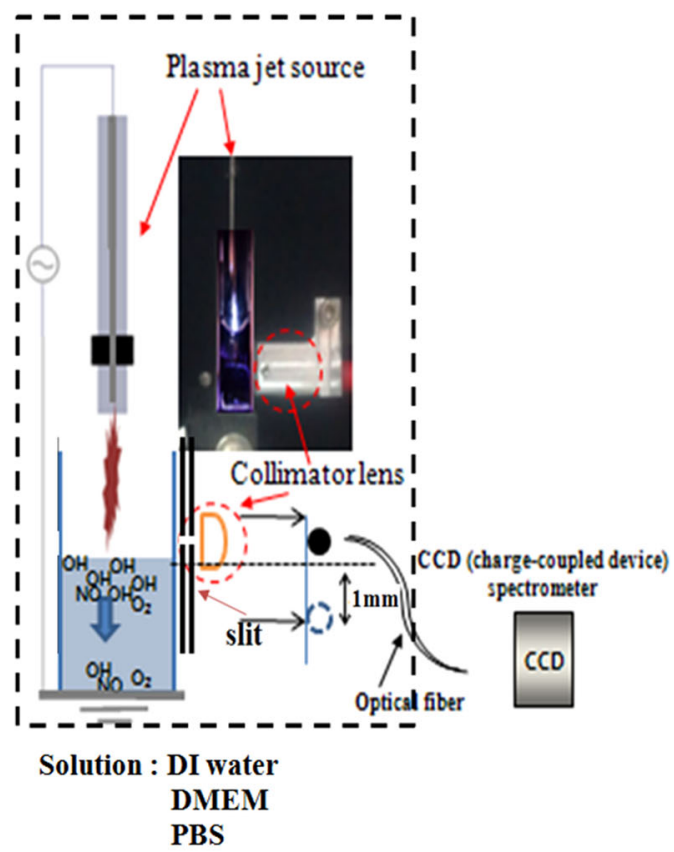

Fig. 3 Schematic experimental setup of ultraviolet absorption spectroscopy below the biosolutions

species inside the biosolution under the argon non-thermal plasma jet bombardment onto the solution.

\section{Results and Discussion}

Electron Temperature and Ion Density Measurement of Argon Non-thermal Plasma Jet

Rare gases such like argon are very general plasma constituents in many applications. Especially, argon can provide useful diagnostics such as electron temperature of nonthermal plasma operating at atmospheric pressure. CR model might be performed for pure Ar gas as long as possible. We have tried to apply the CR model for Ar plasma in contact with biological solution where there are very large amount of water vapor and the plasmaforming gas, since the excited $2 p$ emission lines from Ar atoms are not changed by addition of water molecules and other plasma-forming gases. The electron temperature in Ar plasma in contact with biological solution could be measured accurately by our CR model, since the physical parameters of rate coefficients for Ar gas plasma have been well studied and established [13] for electron temperature measurements. We have also assumed that these physical parameters of rate coefficients for Ar gas at atmospheric pressure can be used even to the environments in contact with biological solutions. Therefore, the CR model for Ar plasma in contact with biological solution could be used for the measurement of electron temperature as in pure Ar gas without any loss of generality. 
A simple atmospheric pressure CR model can be used to determine the electron temperature of non-thermal plasma even operating at atmospheric pressure by using the relative emission intensities of only four argon lines [11]. In this method, a MaxwellBoltzmann distribution for the electron energy distribution function (EEDF) for the nonthermal plasma jet is assumed, and experimental metastable emission data are utilized so that an electron temperature could be obtained. The atmospheric pressure CR model is based on the balance equation of the four excited level densities from any of the $2 p$ levels. Population of the $2 \mathrm{p}$ excited argon levels ( $2 p_{1} \sim 2 p_{10}$ in Paschen notation) mainly occurs by electron collisions with argon atoms in the ground level and with atoms in the metastable levels ( $1 s_{3}$ and $1 s_{5}$ in Paschen notation) [11]. Here the excited Ar atoms to $2 \mathrm{p}$ levels from resonant states $\left(1 s_{2}\right.$ and $\left.1 s_{4}\right)$ and quenching effects caused by the excited atoms from higher $3 p$ levels [13] have been neglected for analytical simplicity since most of emission intensities are caused by electron impact in this experiment. Therefore, the balance equation for a $2 p_{x}$ (Paschen notation) excited level can be written as,

$$
n_{e} n_{g} k_{g, 2 p_{x}}+\sum_{i=3,5} n_{e} n_{1 s_{i}} k_{1 s_{i}, 2 p_{x}}=\sum_{i=5}^{2} n_{2 p_{x}} A_{2 p_{x}, 1 s_{i}},
$$

where $n$ indicates number density $\left(n_{e}\right.$ : electron density, $n_{g}$ : neutral gas density, $n_{1 s}: 1 \mathrm{~s}$ level number density, $n_{2 p}$ : $2 \mathrm{p}$ level number density), $k_{x, y}$ is the rate coefficient for the excitation from level $x$ to $y$, and $A_{y, x}$ is the transition probability form level $y$ to $x$. The subscript $g$ stands for ground level, $e$ for electrons, and $1 \mathrm{~s}_{x}$ and $2 \mathrm{p}_{x}$ are the Paschen notations [11]. In Eq. (2), the rate coefficients $k$ can be calculated according to the following

$$
k\left(T_{e}\right)=\int_{0}^{\infty} \sigma(\varepsilon) \sqrt{\frac{2 \varepsilon}{m}} f\left(\varepsilon, T_{e}\right) d \varepsilon
$$

where $\varepsilon$ is the energy, $m$ is the electron mass, $f$ is the Maxwell-Boltzmann EEDF, $\sigma$ is the cross section of the collision between electrons and the particles [11]. The relative number density for specified wavelength was represented by the emission intensities observed to the spectrometer. For the reason, the $n_{x}(x=1 \mathrm{~s}, 2 \mathrm{p})$ in Eq. (3) can be written as,

$$
n_{x} \approx \frac{I_{x, y} \lambda_{x, y}}{A_{x, y}},
$$

where $\mathrm{x}$ and $\mathrm{y}$ represent upper and lower energy levels, $\mathrm{I}$ is the relative intensity, and $\lambda$ the specified wavelength [11]. Eq. (4) can be used in Eq. (2) to replace $\mathrm{n}_{2 p x}$ and Eq. (2) can be written as follows

$$
n_{e}=\frac{\sum_{i=5}^{2} n_{2 p_{x}} A_{2 p_{x}, 1 s_{i}}}{n_{g} k_{g, 2 p_{x}}+\sum_{i=3,5} n_{1 s_{i}} k_{1 s_{i}, 2 p_{x}}}
$$

This equation can be represented by any $2 p_{x}$ level (indicated as $2 \mathrm{p}_{2}, 2 \mathrm{p}_{3}, 2 \mathrm{p}_{6}$ and $2 \mathrm{p}_{10}$ ). It is noted here that these $2 \mathrm{p}$ metastable Ar emission lines are $696.5 \mathrm{~nm}\left(2 \mathrm{p}_{2} \rightarrow 1 \mathrm{~s}_{5}\right)$, $706.7 \mathrm{~nm}\left(2 \mathrm{p}_{3} \rightarrow 1 \mathrm{~s}_{5}\right), 922.4 \mathrm{~nm}\left(2 \mathrm{p}_{6} \rightarrow 1 \mathrm{~s}_{2}\right)$, and $912.3 \mathrm{~nm}\left(2 \mathrm{p}_{10} \rightarrow 1 \mathrm{~s}_{5}\right)$. This electron density $n_{e}$ is the same for four $2 \mathrm{p}_{x}$ levels. Therefore, we can couple these equations as followings [11], 


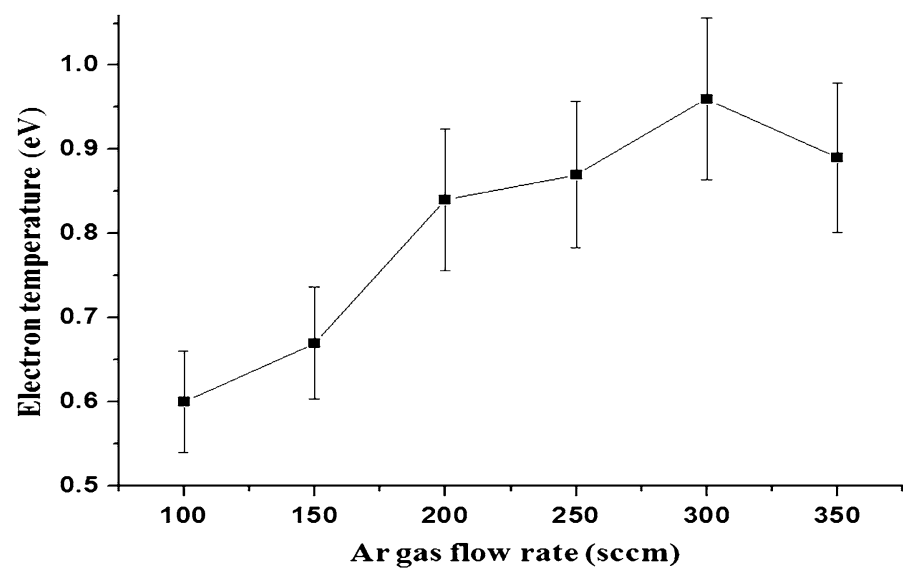

Fig. 4 Electron temperatures of Ar non-thermal plasma jet at $2 \mathrm{~mm}$ above the biosolution versus the argon flow rates

$$
\begin{aligned}
& \frac{\sum_{i=5}^{2} n_{2 p_{a}} A_{2 p_{a}, 1 s_{i}}}{n_{g} k_{g, 2 p_{a}}+\sum_{i=3,5} n_{1 s_{i}} k_{1 s_{i}, 2 p_{a}}}=\frac{\sum_{i=5}^{2} n_{2 p_{b}} A_{2 p_{b}, 1 s_{i}}}{n_{g} k_{g, 2 p_{b}}+\sum_{i=3,5} n_{1 s_{i}} k_{1 s_{i}, 2 p_{b}}} \\
& \frac{\sum_{i=5}^{2} n_{2 p_{c}} A_{2 p_{c}, 1 s_{i}}}{n_{g} k_{g, 2 p_{c}}+\sum_{i=3,5} n_{1 s_{i}} k_{1 s_{i}, 2 p_{c}}}=\frac{\sum_{i=5}^{2} n_{2 p_{d}} A_{2 p_{d}, 1 s_{i}}}{n_{g} k_{g, 2 p_{d}}+\sum_{i=3,5} n_{1 s_{i}} k_{1 s_{i}, 2 p_{d}}}
\end{aligned}
$$

The electron temperature $T_{e}$ of any range value is replaced by $k$ and Eqs. (6) and (7) can be solved with respect to metastable number densities $n_{1 s_{3}}$ and $n_{1 s_{5}}$. For example, we can obtain two equations for the electron density $n_{e-a}\left(T_{e}\right)$ of $2 p_{a}$ excited level and $n_{e-c}\left(T_{e}\right)$ of $2 p_{c}$ excited level that are dependent on the electron temperature $T_{e}$. For obtaining the electron temperature of plasma jet, we have to find the specific electron temperature being satisfied with the condition,

$$
n_{e-a}\left(T_{e}\right)=n_{e-c}\left(T_{e}\right)
$$

This calculation process for obtaining the electron temperature can be easily processed using simple Matlab programming. The electron temperature for the argon non-thermal plasma jet operating at the atmospheric pressure with low electrical power of $4.9 \mathrm{~W}$ and driving frequency of $35 \mathrm{kHz}$ could be measured by optical emission spectroscopy under the various Ar gas flows ranged from 100 to $350 \mathrm{sccm}$ based on the atmospheric pressure CR model.

Figure 4 shows the measured electron temperatures for the argon non-thermal plasma jet versus the gas flow rates ranged from 100 to $350 \mathrm{sccm}$, obtained by the atmospheric pressure CR model with the four $2 p$ metastable Ar emission lines. It is noted that these electron temperatures $k T e$ have been measured at the region of $2 \mathrm{~mm}$ above the biosolution surface. It is found that electron temperature $k T e$ has been increased up to about $1 \mathrm{eV}$ as the argon gas flow rate is increased to $300 \mathrm{sccm}$, and it is slowly decreased beyond $300 \mathrm{sccm}$ and reached to $0.9 \mathrm{eV}$ at $350 \mathrm{sccm}$. It is noted that the propagating speed, $v_{p}$, of plasma jet is given by $v_{p}=v^{2} / u_{n}$ [14] based on the diffusion wave-packet model, where $v$ is the ion sound or Bohm's velocity and $u_{n}$ is density gradient gas speed. Here it is noted that $v_{p}$ could be estimated as $\sim 20 \mathrm{~km} / \mathrm{s}$ with $u_{n}$ of $\sim 10 \mathrm{~m} / \mathrm{s}$ [15], so that 
the $v$ is estimated to be $0.4 \times 10^{3} \mathrm{~m} / \mathrm{s}$. From the equation of $v \approx \sqrt{\frac{k T_{e}}{M}}$, where $M$ is atomic mass of $\operatorname{Ar}\left(M=6.63 \times 10^{-26} \mathrm{~kg}\right)$, the electron temperature $k T e$ could be also obtained by about $1 \mathrm{eV}$. This electron temperature obtained from the ion sound speed $v$ and the plasma propagation speed $v_{p}$ is in good agreement with that obtained by atmospheric pressure CR model. There are, of course, uncertainties surrounding the use of a CR model that considers only argon to analyse a plasma jet impinging on a solution. However, because the measurements were taken $2 \mathrm{~mm}$ above the substrate, and because the electron temperature obtained from the ion sound speed and plasma propagation speed was in agreement, we believe that the temperatures are reliable.

The plasma ion density $n_{i}$ can be obtained from measurements of the plasma ion collector current $I_{i}$, after passing through the floating mesh, which can be measured by [16]

$$
n_{i}(r)=\frac{I_{i}}{2 \pi e v \sigma^{2}} \exp \left(-\frac{r^{2}}{2 \sigma^{2}}\right),
$$

where $e$ is electron charge, $v$ is ion sound velocity, $\sigma$ is the width of the Gaussian profile for emission intensity distribution of plasma jet, which is $6 \mathrm{~mm}$ in this plasma jet, and $r$ is the radial distance from the center. The ion density has been measured by RMS value of ion current at the radial distance of $r=0$ in this experiment. At gas flow ranges between 150 and $300 \mathrm{sccm}$, the ion densities are measured to be about $1 \times 10^{13} \mathrm{~cm}^{-3}$.

Optical Emission Spectroscopy of Non-thermal Plasma Jet Inside the Deionised (DI) Water

Especially, optical emission spectroscopy (OES) inside biosolutions is extremely useful for non-thermal plasma jet bombarding onto the surface since the information's for induced ROS and their spatial distribution characteristics could be obtained from these data. Atmospheric pressure non-thermal plasma could generate various kinds of reactive oxygen species inside the biosolutions during their interactions on the surface. Plasma-induced ultraviolets have been generated by reactive species of nitric oxide (NO) [17, 18], superoxide anion $\left(\mathrm{O}_{2}{ }^{*^{-}}\right)$[19], hydrogen peroxide $\left(\mathrm{H}_{2} \mathrm{O}_{2}\right)$ [20], and hydroxyls $(\mathrm{OH})[4,10$, 18, 21-23] on boundary surface of plasma and biosolutions. These ultraviolets, whose wavelength ranged from $210 \mathrm{~nm}(6 \mathrm{eV})$ to $309 \mathrm{~nm}(4.2 \mathrm{eV})$, propagate into solutions below more than $2 \mathrm{~mm}$ to excite the water molecules and dissociate them to induce ROS generation inside the biosolution. This is a mechanism for the ROS generation inside the solution.

Figure 5 shows the visual confirmation of $\mathrm{OH}$ radical species generation inside the deionized (DI) water by bombardment of non-thermal plasma jet onto the water surface, in which vinyl filter has been placed just below the water surface, with (left hand side) and without (right hand side) inclusion of terephthalic acid (TA) [24]. The vinyl filter could pass only ultraviolet lights from 260 to $320 \mathrm{~nm}$, however, the plasma particles are prohibited from this filter. It is found that the hydroxyl $\mathrm{OH}$ radical species could be generated inside the DI water since the color of the DI water including TA is changed to blue in this experiment. The water $\mathrm{H}_{2} \mathrm{O}$ molecules could be excited to higher energy levels $\mathrm{H}_{2} \mathrm{O}^{*}$ and dissociated into $\mathrm{OH}$ by these successive UV exposures to them, whose wavelengths are mainly $309 \mathrm{~nm}$ which are about $4 \mathrm{eV}$, i.e., $\mathrm{UV}+\mathrm{H}_{2} \mathrm{O} \rightarrow \mathrm{H}_{2} \mathrm{O}^{*}$ and $\mathrm{UV}+\mathrm{H}_{2} \mathrm{O}^{*} \rightarrow \mathrm{OH}+\mathrm{H}$. Hence we could think that the plasma-induced ultraviolet lights on the biosolution play a major role for production of reactive oxygen species inside the biosolutions by propagation into them. 
Fig. 5 Generation of $\mathrm{OH}$ radical species by non-thermal plasma in deionized (DI) water with inclusion of terephthalic acid (TA)

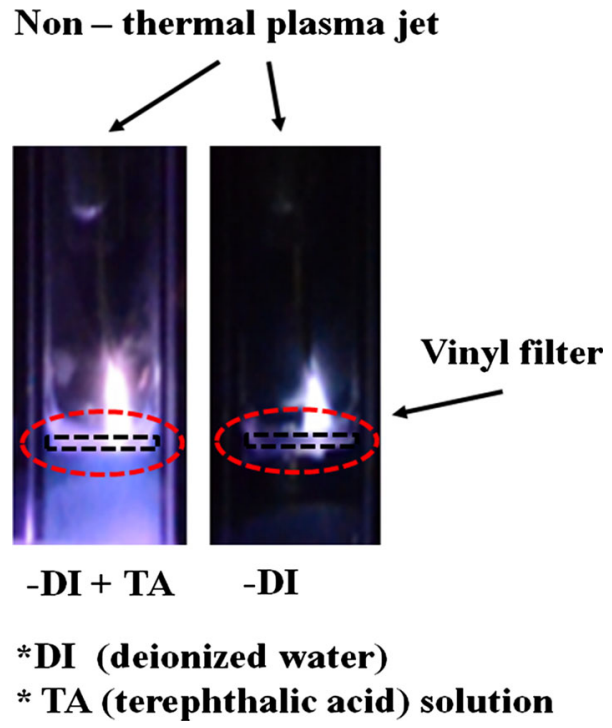

Figure 6 shows the optical emission spectrum measured by CCD spectrometer with optical fiber at the $2 \mathrm{~mm}$ above the deionised (DI) biosolution surface (a), and $2 \mathrm{~mm}$ below the the DI biosolution surface (b). From the result of spectrum of Fig. 6a, the Ar emission lines between 700 and $900 \mathrm{~nm}$ are shown to be dominantly appeared and it is found that the $309 \mathrm{~nm}$ emitted from the hydroxyl $\mathrm{OH}$ radical [4, 8, 18, 21-23] species, $224 \mathrm{~nm}$ from the nitric oxide NO [17, 18], the emission lines $245 \mathrm{~nm}$ from the reactive species of superoxide anion $\mathrm{O}_{2}{ }^{--}$[19], and the emission lines from $\mathrm{N}_{2}$ second positive system (C3Пu-B3Пg) ranged from 320 to $380 \mathrm{~nm}$ are strongly appeared just above the biosolution surface. While as seen in Fig. 6b, as moved $2 \mathrm{~mm}$ downward into the DI biosolution surface, the emission lines of $224 \sim 280 \mathrm{~nm}$ from the nitric oxide NO [17, 18], the $245 \mathrm{~nm}$ from the reactive species of superoxide anion $\mathrm{O}_{2} *^{-}$[19] are shown to be decreased and disappeared. Also it is found that the $309 \mathrm{~nm}$ emitted from the hydroxyl OH radical species gets stronger in the biosolution than that above the solution. It is also noted from the Fig. $6 \mathrm{a}$ and $\mathrm{b}$ that the both lines of 777 and $852 \mathrm{~nm}$ inside the DI biosolution, which are emitted from the $\mathrm{O}_{2}$ first positive system, including the emission lines of Ar are getting weaker than those above the solution.

Density of Hydroxyl OH Radical Species and Its Influence on the Biological Cells

The density of the hydroxyl radical species has been investigated at the region of $2 \mathrm{~mm}$ above the interfacial solution surface [10], where the UV passing length is $3 \mathrm{~mm}$, as well as inside the biosolution whose spatial passing length is $10 \mathrm{~mm}$, by the ultraviolet absorption spectroscopy in Ar gas flow ranged from 100 to $350 \mathrm{sccm}$ in argon non-thermal plasma jet. At this time the position of the deuterium lamp and plano-convex lens system have been fixed during the measurement. For the measurement of $\mathrm{OH}$ radical species at the different position inside the biosolution, the detector system could be moved down from $2 \mathrm{~mm}$ below water surface with spatial resolution of $1 \mathrm{~mm}$. 

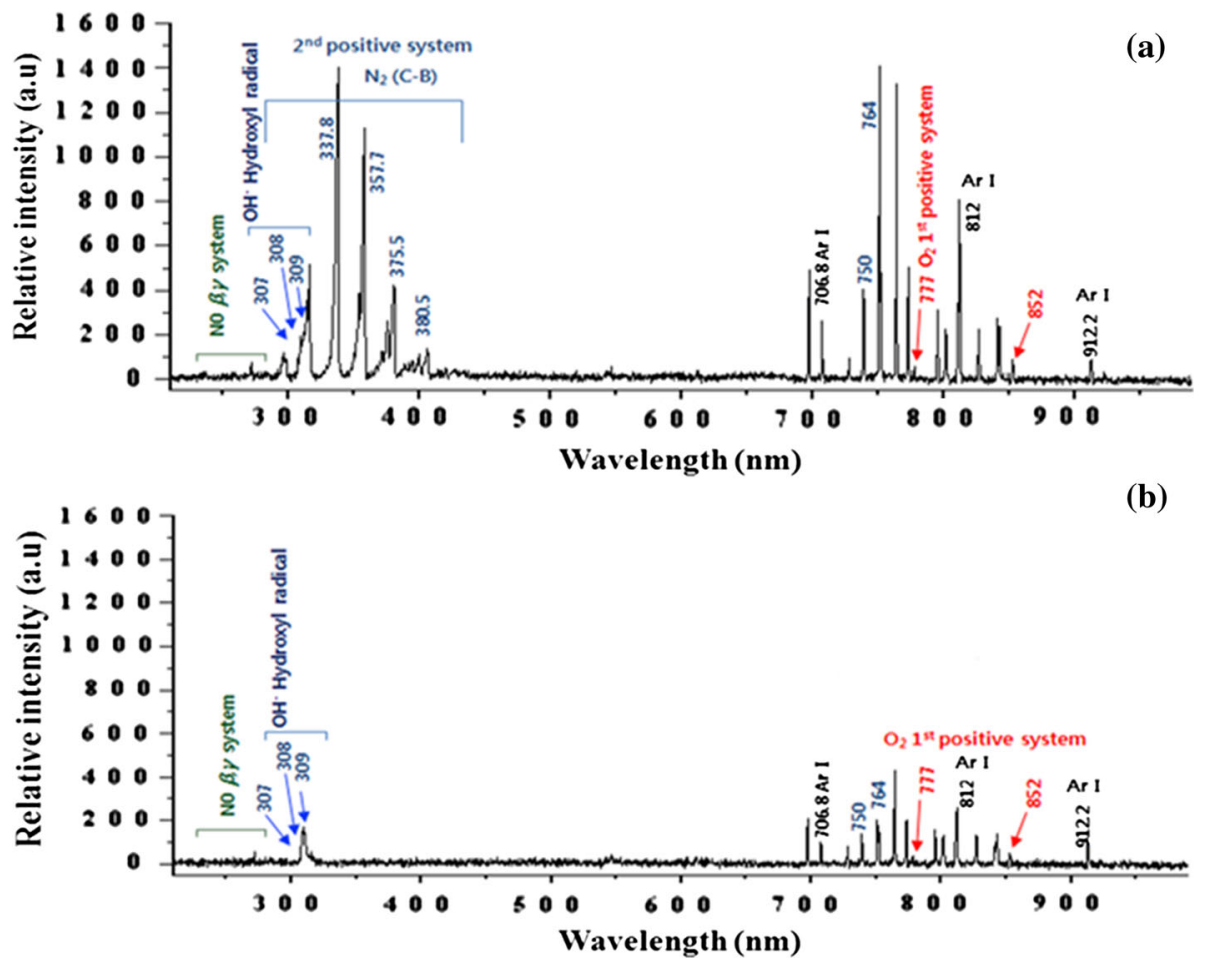

Fig. 6 Optical emission spectrum measured by CCD spectrometer with optical fiber at 2 mm above DI water (a), and $2 \mathrm{~mm}$ below the DI water surface (b)

Figure 7 shows the UV emission and absorption spectral profiles caused by the $\mathrm{OH}$ radical species, at the region of $2 \mathrm{~mm}$ above the DI water surface (a) and inside them (b), respectively, versus the wavelengths. Also it is noted that reference UV lamp $\left(I_{o}\right)$ without non-thermal plasma has been shown at the upper line (black) and ROS emission profiles with non-thermal plasma have been shown at the bottom lines (blue), respectively, in Fig. 7a and $\mathrm{b}$. It is especially noted that the $\mathrm{OH}$ absorption profiles are strongly appeared around the $306 \sim 309 \mathrm{~nm}$ at the round-dotted region (red) for both regions of Fig. 7a and b. It is also noted from Fig. 7 that the absorption profiles for the other ROS species such as NO $(226 \mathrm{~nm})$ and $\mathrm{O}_{2}{ }^{--}(245 \mathrm{~nm})$ are shown to be very small above the DI surface of Fig. 6a, while they are disappeared inside the DI water of Fig. $7 \mathrm{~b}$. The absorption profiles of hydroxyl $\mathrm{OH}$ radical species (red) have been shown to be maximum in both Fig. 7a and $\mathrm{b}$ around the wavelength of $309 \mathrm{~nm}$ under the non-thermal plasma bombardment and the reference lamp is turned on. It is noted that the UV transmission ratio $I_{v}$ to incident one $I_{o}$ around $309 \mathrm{~nm}$ could be obtained by comparison between the emission profiles from the reference deuterium lamp (black) and the absorption profiles of $\mathrm{OH}$ radical species (red), as shown in the Fig. 7a. This transmission ratio $\left(I_{v} / I_{o}\right)$ for $\mathrm{OH}$ radical species has been measured to be 0.75 and 0.32 , respectively, for on the surface and inside the biosolution, respectively, where the intensity reaches the maximum value in Fig. $7 \mathrm{a}$ and b. The emission and absorption spectra of $\mathrm{OH}$ in water are observed to be basically the same as those of $\mathrm{OH}$ in the gas phase, in this experiment, which are mainly occurred at $309 \mathrm{~nm}$. We 

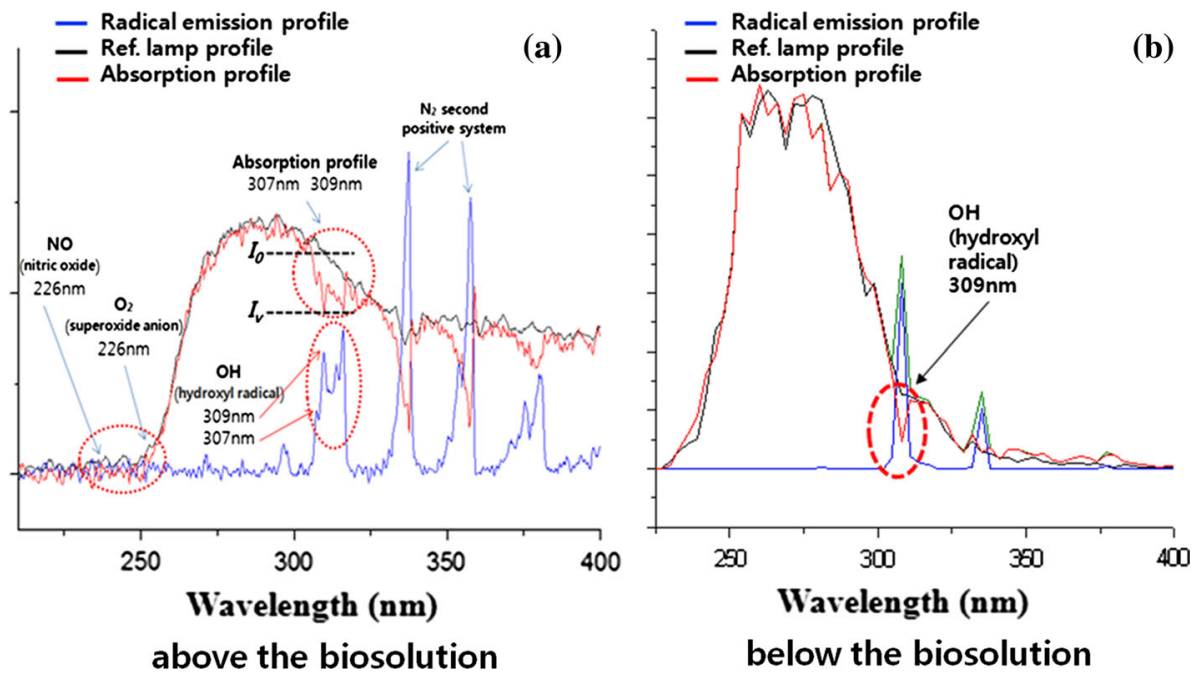

Fig. 7 UV emission and absorption profiles caused by the ROS including OH species, above the deionized (DI) (a) and below it (b), versus the wavelengths

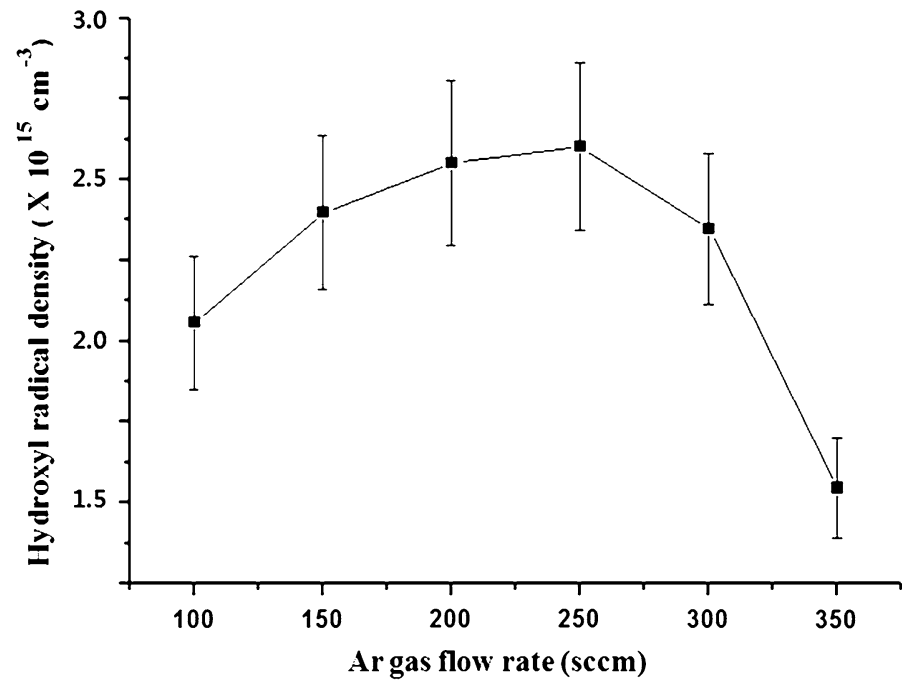

Fig. 8 Density of hydroxyl $\mathrm{OH}$ radical species at the $2 \mathrm{~mm}$ above the deionized (DI) water surface, versus the $\mathrm{Ar}$ gas flow rates ranged from $100 \mathrm{sccm}$ to $350 \mathrm{sccm}$

emphasize that we could measure $\mathrm{OH}$ density inside the biological solutions for $\mathrm{OH}$ region of $x=10 \mathrm{~mm}$ using the UV absorption methods as in the gas phase above the DI water for $\mathrm{OH}$ region of $x=3 \mathrm{~mm}$.

Figure 8 shows the density of hydroxyl $\mathrm{OH}$ radical species at the region of $2 \mathrm{~mm}$ above the DI water, versus the Ar gas flow rates ranged from 100 to $350 \mathrm{sccm}$, under the low electrical power of $4.9 \mathrm{~W}$ and driving frequency of $35 \mathrm{kHz}$. It is noted that these 


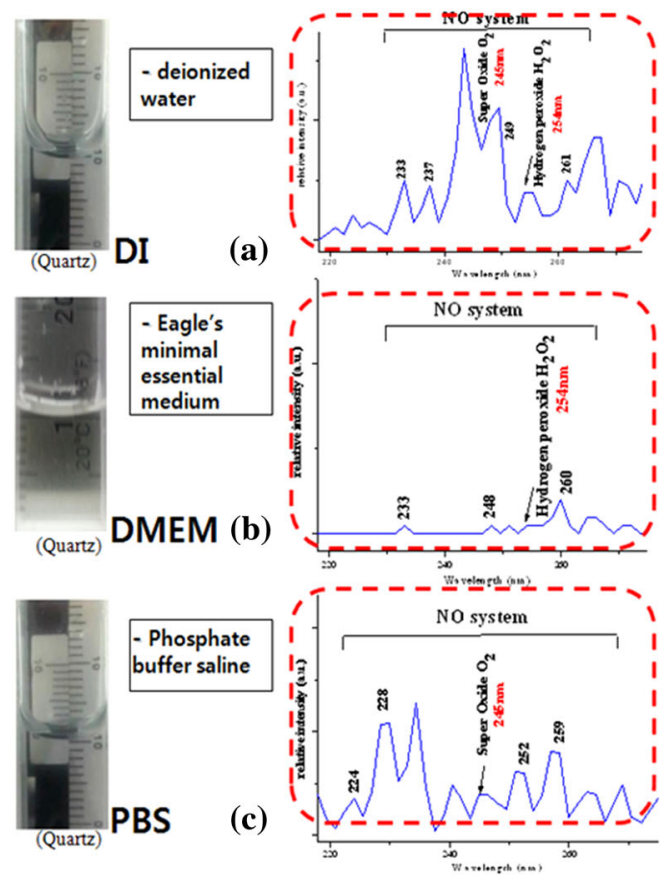

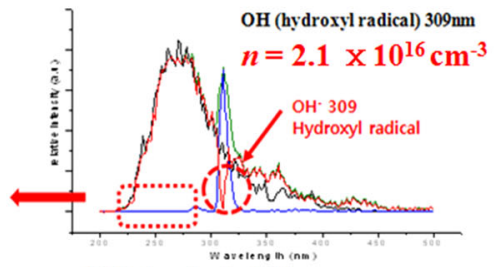
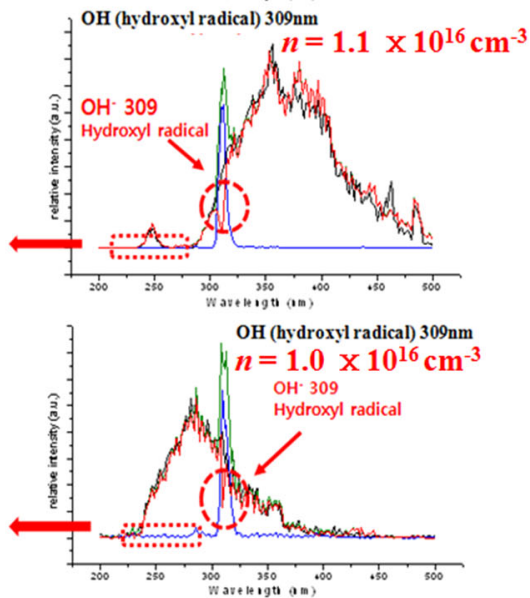

Fig. 9 Density of $\mathrm{OH}$ radical species along with the $\mathrm{OH}$ absorption profiles in deionized (DI) water (a), Dulbecco's modified eagle medium (DMEM) $(\mathbf{b})$, and $1 \times$ phosphate buffered saline $(\mathrm{PBS})(\mathbf{c})$ by ultraviolet absorption spectroscopy. As seen in the magnified view images of dotted square region, the ROS intensities from $\mathrm{NO}, \mathrm{O}_{2}^{*-}$, and $\mathrm{H}_{2} \mathrm{O}_{2}$ are quite small in comparison with those for $\mathrm{OH}$ radical species inside the solutions

measurement values are within $5 \%$ error ranges. For the gas flow rate of around $200-250 \mathrm{sccm}$, it is found that the density of hydroxyl $\mathrm{OH}$ radical species reaches the maximum value of $2.6 \times 10^{15} \mathrm{~cm}^{-3}$. It is also noted that this density of $\mathrm{OH}$ radical species has been rapidly decreased to $1.5 \times 10^{15} \mathrm{~cm}^{-3}$ as the gas flow rate is increased to $350 \mathrm{sccm}$ in this experiment. These $\mathrm{OH}$ densities are in relatively good agreement with other results of $(0.3 \sim 7.5) \times 10^{15} \mathrm{~cm}^{-3}$ reported by other groups under the low contents of water molecules $<3 \%$ in their employed gases of $\mathrm{He}, \mathrm{N}_{2}$, and $\mathrm{N}_{2} / \mathrm{O}_{2}$ mixtures $[4,21,22]$. They have used a microwave frequency of $2.45 \mathrm{GHz}[21,22]$ and RF frequency of $13.56 \mathrm{MHz}$ [18] with relatively high power greater than $100 \mathrm{~W}$. However, we have used the low driving frequency of $35 \mathrm{kHz}$ and low electrical power of $4.9 \mathrm{~W}$ in this experiment for the production of $\mathrm{OH}$ radical species inside the DI biosolutions by bombardment of the non-thermal atmospheric pressure plasma onto the DI water with Ar gas for various ranges of gas flow rates.

The density of the $\mathrm{OH}$ radical species inside the various biosolutions have been investigated when the Ar non-thermal plasma jet has been bombarded onto their surface. Figure 9 shows the emission and absorption profiles of the $\mathrm{OH}$ radical species inside the deionized (DI) water (a), Dulbecco's modified eagle medium (DMEM) (b), and 1× phosphate buffered saline (PBS) (c) when the non-thermal plasma jet has been bombarded onto these biosolution surfaces. These emission and absorption optical signals have been measured at $2 \mathrm{~mm}$ below the biosolutions. It is noted here for the wavelength ranges between the 220 and $260 \mathrm{~nm}$ inside the biosolutions that there are emission profiles of 


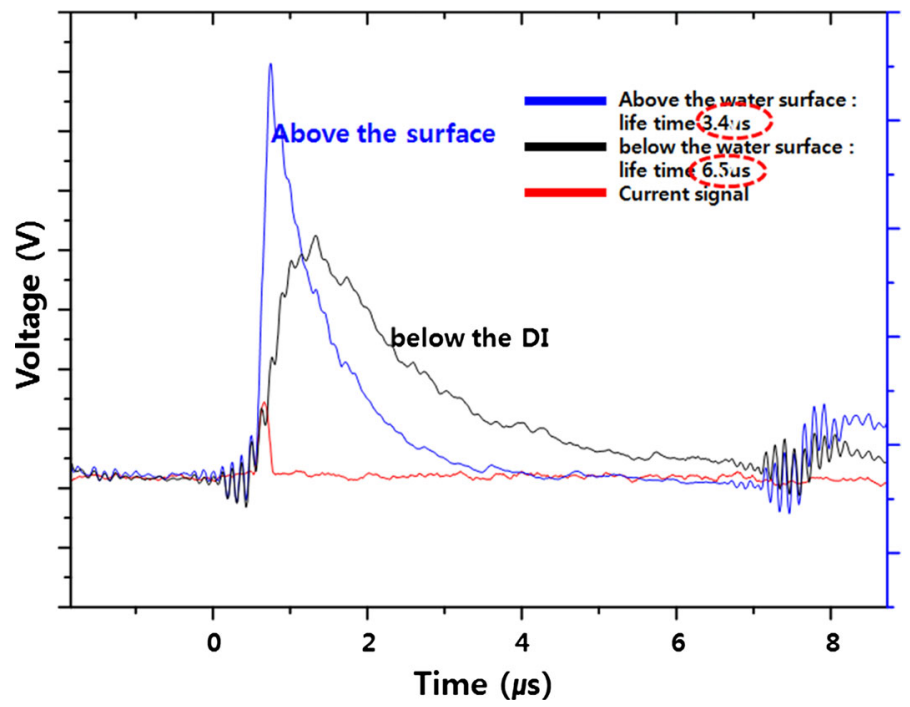

Fig. 10 Temporal emission behaviors of $309 \mathrm{~nm}$ for $\mathrm{OH}$ radical species above and below deionized (DI) water. It is noted that the life times for $\mathrm{OH}$ species are 3.2 and $6.5 \mu$ s above the DI water and below it, respectively

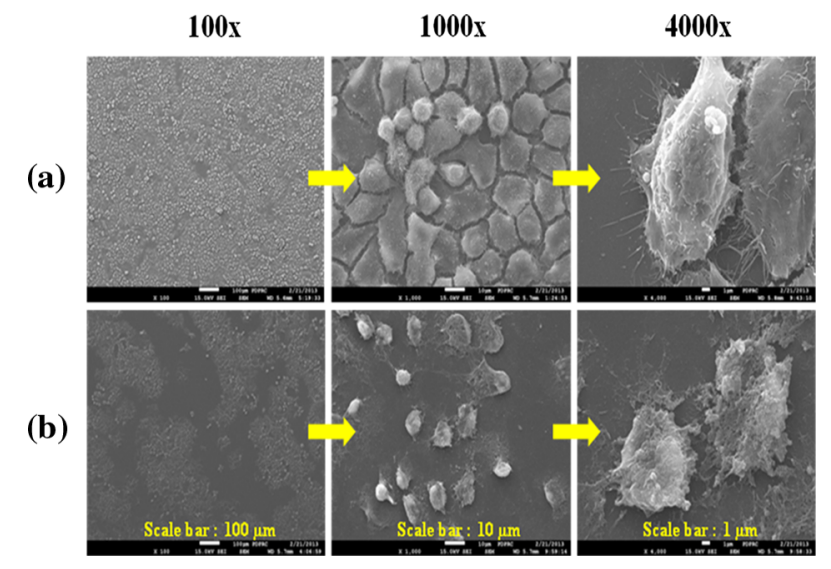

Fig. 11 Scanning electron microscope images of the control (a) and the plasma treated (b) lung cancer cells (H460) with different magnification. Ar non-thermal plasma is exposed to DMEM surface for $1 \mathrm{~min}$, and cells are located $2 \mathrm{~mm}$ below the surface. The morphological changes should be induced by the hydroxyl $\mathrm{OH}$ radicals, since only hydroxyl $\mathrm{OH}$ radical density is measured high to be $1.1 \times 10^{16} \mathrm{~cm}^{-3}$ at $2 \mathrm{~mm}$ below the DMEM surface

$224 \mathrm{~nm}$ for the nitric oxide $\mathrm{NO}[17,18], 245 \mathrm{~nm}$ for the superoxide anion $\mathrm{O}_{2}{ }^{*^{-}}$[19], and $254 \mathrm{~nm}$ for the hydrogen peroxide $\mathrm{H}_{2} \mathrm{O}_{2}$ [20] as seen in the magnified view images of dotted square region of Fig. 9. However, their intensities are quite small in comparison with those for $\mathrm{OH}$ radical species inside the biosolutions. It is also noted in this experiment that the densities of the $\mathrm{OH}$ radical species inside the DI water, DMEM, and PBS are measured to be about $2.1 \times 10^{16}, 1.1 \times 10^{16}$, and $1.0 \times 10^{16} \mathrm{~cm}^{-3}$, respectively, at $2 \mathrm{~mm}$ downstream the surface during non-thermal plasma operation under Ar gas flow $200 \mathrm{sccm}$. 
Also we have investigated the life times for $\mathrm{OH}$ radical species above the DI water surface and inside it, respectively. Figure 10 shows the temporal emission behaviors of $309 \mathrm{~nm}$ for $\mathrm{OH}$ radical species above the DI water and below it under Ar gas flow of $200 \mathrm{sccm}$, along with the electrical current signals. It is noted that the hydroxyl $\mathrm{OH}$ radical species above the DI has been generated simultaneously with respect to on-time of electrical current, while the $\mathrm{OH}$ radical species below the DI are increased slowly with respect to on-time of electrical current. It is noted that the life times for $\mathrm{OH}$ species are found to be $3.2 \mu \mathrm{s}$ above the DI water and $6.5 \mu \mathrm{s}$ below that, respectively, with respective rising time of $\sim 100$ and $\sim 200 \mathrm{~ns}$, in this experiment. Also, the falling time for $\mathrm{OH}$ radical species generated inside the DI water is measured to be about $6 \mu$ s, which is two times longer than $2.5 \mu$ s above the water. These longer life times and falling times of $\mathrm{OH}$ inside the solutions are main reasons for the higher $\mathrm{OH}$ density since the $\mathrm{OH}$ could be generated by two main pathways of $\mathrm{UV}+\mathrm{H}_{2} \mathrm{O}^{*} \rightarrow \mathrm{OH}$ and $\mathrm{UV}+\mathrm{H}_{2} \mathrm{O}_{2}{ }^{*} \rightarrow \mathrm{OH}$ inside the water in comparison with air environment, which results in higher density of $\mathrm{OH}$ than air environment. The detail investigations for this will be published soon elsewhere.

We have prepared the lung cancer cell (H460), which is immersed and adhered on the inner bottom surface of petri dish with DMEM media, whose depth is $2 \mathrm{~mm}$ from the surface for the investigation of influence of hydroxyl $\mathrm{OH}$ density on this cell. Figure 11 shows the morphology for the control lung cancer cell (H460), magnified by $100 \times$, $1,000 \times$, and $4,000 \times(\mathrm{a})$, and the morphology changes in the plasma treated lung cancer cell (H460), magnified by $100 \times, 1,000 \times$, and $4,000 \times$, for $1 \mathrm{~min}$ exposure to DMEM surface, where it is noted that the hydroxyl $\mathrm{OH}$ density is measured to be $1.1 \times 10^{16} \mathrm{~cm}^{-3}$ at the adhered cell location of $2 \mathrm{~mm}$ downstream (b), respectively. It has been observed that smooth flat surfaces for control lung cancer cells, as in Fig. 11a, has been collapsed and crushed with shrunken surfaces as shown in Fig. 11b by the mutual interaction of hydroxyl $\mathrm{OH}$ species with lung cancer cells, where the hydroxyl $\mathrm{OH}$ radicals are produced inside the solution by the plasma treatment on the solution surface. With adjustment of experimental conditions, it is found that the threshold $\mathrm{OH}$ density for apoptosis of $\mathrm{H} 460$ cells inside the DMEM is about $0.3 \times 10^{16} \mathrm{~cm}^{-3}$ under 1 min plasma exposure, which may be followed by mitochondrial damages as reported previously [25].

\section{Conclusions}

Non-thermal atmospheric pressure plasma jet has been developed for biomedical research fields with less electrical power operation of $4.9 \mathrm{~W}$ and driving frequency of $35 \mathrm{kHz}$. This paper contains the very informative data for describing the basic properties of non-thermal plasma jet such as the electron temperature on the biosolution surface as well as density of hydroxyl $\mathrm{OH}$ radical species inside the biosolutions during plasma bombardment onto the surface. A simple CR model has been employed in this experiment to determine the electron temperature of non-thermal plasma jet operating at atmospheric pressure by using the relative emission intensities of only four $2 \mathrm{p} \mathrm{Ar}$ lines. It is noted that the electron temperatures have been measured at the region of $2 \mathrm{~mm}$ above the biosolution surface. It is found that electron temperature has been measured to be about $0.8 \sim 1 \mathrm{eV}$ as the argon gas flow rate is increased from 200 to $300 \mathrm{sccm}$, and it is slowly decreased beyond $300 \mathrm{sccm}$ and reached to $0.9 \mathrm{eV}$ at $350 \mathrm{sccm}$.

Especially, when non-thermal plasma jet is operating on the biosolution surface, we have investigated that various reactive oxygen species (ROS) and reactive nitrogen species 
(RNS) have been generated near the biosolution surface as well as inside them. The emission lines of $224 \sim 280 \mathrm{~nm}$ from the nitric oxide NO, the $245 \mathrm{~nm}$ from the superoxide anion $\mathrm{O}_{2}{ }^{-,}$, and $\mathrm{N}_{2}$ second positive system (C3Пu-B3Пg) emission lines ranged from 320 to $380 \mathrm{~nm}$ are shown to be drastically decreased inside the biosolution of $2 \mathrm{~mm}$ downward its surface. However, it is found that the $309 \mathrm{~nm}$ emission from the hydroxyl OH radical species gets stronger in the biosolution than that above the solution.

It is found at the region of $2 \mathrm{~mm}$ above the solution surface that the density of hydroxyl $\mathrm{OH}$ radical species reaches the maximum value of $2.6 \times 10^{15} \mathrm{~cm}^{-3}$ for the gas flow rate of around 200-250 sccm in this experiment. Also the density of the $\mathrm{OH}$ radical species inside the various biosolutions have been investigated when the argon non-thermal plasma jet has been bombarded onto their surface. The densities of the $\mathrm{OH}$ radical species inside the DI water and DMEM are also measured to be about $2.1 \times 10^{16}$ and $1.1 \times 10^{16} \mathrm{~cm}^{-3}$, respectively, at $2 \mathrm{~mm}$ downstream the surface under $\mathrm{Ar}$ gas flow $200 \mathrm{sccm}$ in this experiment. The $\mathrm{OH}$ density inside the PBS solution has been measured to be about $1.0 \times 10^{16} \mathrm{~cm}^{-3}$ at $2 \mathrm{~mm}$ downstream the surface during non-thermal plasma operation. It is found that the hydroxyl $\mathrm{OH}$ radical species above the DI water has been generated simultaneously with respect to on-time of electrical current, while the $\mathrm{OH}$ radical species below the DI are increased slowly with respect to on-time of electrical current. The life times for $\mathrm{OH}$ species are measured to be $3.2 \mu$ s above the DI water and $6.5 \mu$ s below that, respectively, with respective rising time of $\sim 100$ and $\sim 200 \mathrm{~ns}$. Also, the falling time for $\mathrm{OH}$ radical species generated inside the DI water is measured to be about 2.3 times longer than that above the water. These characteristics of longer $\mathrm{OH}$ life time inside the DI water might result in higher density for $\mathrm{OH}$ radical species in comparison with that above it.

It has been observed that smooth flat surfaces for control lung cancer cells has been collapsed and crushed with shrunken surfaces by the mutual interaction of hydroxyl $\mathrm{OH}$ radicals, where these $\mathrm{OH}$ radical species are produced inside the solution by the plasma treatment on the solution surface. It is found in this experiment that the threshold $\mathrm{OH}$ density for apoptosis of H460 cells inside the DMEM is about $0.3 \times 10^{16} \mathrm{~cm}^{-3}$ under 1 min plasma exposure.

Furthermore, it is necessary and essential to investigate the standard reference criterion of hydroxyl $\mathrm{OH}$ critical density for various kinds of cancer cells to induce their apoptosis when we will use the non-thermal atmospheric pressure plasma for healthcare applications throughout the future works.

Acknowledgments This work has been supported by a National Research Foundation of Korea (NRF) grant funded by the Ministry of Science, ICT and Future Planning (MSIP) (NRF-2010-0027963), (20120009639) and by the Korea Institute of Energy Technology Evaluation and Planning (KETEP) granted from the Ministry of Trade, Industry \& Energy (No. 20113020010080) of Korea Government. This work is also partially supported by Kwangwoon University 2014.

Open Access This article is distributed under the terms of the Creative Commons Attribution License which permits any use, distribution, and reproduction in any medium, provided the original author(s) and the source are credited.

\section{References}

1. Hong YC, Uhm HS, Yi WJ (2008) Atmospheric pressure nitrogen plasma jet: observation of striated multilayer discharge patterns. Appl Phys Lett 93:051504

2. Bandyopadhyay U, Das D, Banerjee RK (1999) Reactive oxygen species: oxidative damage and pathogenesis. Current Sci 77:658 
3. Reactive Oxygen Species (ROS), http://users.rcn.com/jkimball.ma.ultranet/BiologyPages/R/ROS.html

4. Dorn HP, Neuroth R, Hofzumahaus A (1995) Investigation of OH absorption cross sections of rotational transitions in the $\mathrm{A} 2 \mathrm{~S}+, \mathrm{v} 0=0 \mathrm{X} 2 \tilde{\mathrm{O}}$, v00 $=0$ band under atmospheric conditions: implications for tropospheric long-path absorption measurements. J Geophys Res 100:7397

5. Oehmigen K, Winter J, Hähnel M, Wilke C, Brandenburg R, Weltmann KD, von Woedtke T (2011) Estimation of possible mechanisms of escherichia coli inactivation by plasma treated sodium chloride solution. Plasma Process Polym 8:904-913

6. Kong MG, Kroesen G, Morfill GE, Nosenko T, Shimizu T, van Dijk J, Zimmerman JL (2009) Plasma medicine: an introductory review. New J Phys 11:115012

7. Dobrynin D, Fridman G, Friedman G, Fridman A (2009) Physics and biological mechanisms of direct plasma interaction with living tissue. New J Phys 11:115020

8. Alkawareek MY, Algwari QT, Laverty G, Gormanl SP, Graham WG, O'Connell D, Gilmore BF (2012) Eradication of Pseudomonas aeruginosa biofilms by atmospheric pressure non-thermal plasma. PLoS ONE 7(8):e44289

9. Vandamme M, Robert E, Lerondel S, Sarron V, Ries D, Dozias S, Sobilo J, Gosset D, Kieda C, Legrain B, Pouvesle JM, Pape AL (2012) ROS implication in a new antitumor strategy based on non-thermal plasma. Int J Cancer 130:2185

10. Hong YJ, Nam CJ, Song KB, Cho GS, Uhm HS, Choi DI, Choi EH (2012) Measurement of hydroxyl radical density generated from the atmospheric pressure bioplasma jet. JINST 7:C03046

11. Mariotti D, Shimizu Y, Sasaki T, Koshizaki N (2006) Method to determine argon metastable number density and plasma electron temperature from spectral emission originating from four $4 \mathrm{p}$ argon levels. Appl Phys Lett 89:201502

12. Dieke GH, Crosswhite HM (1961) The ultraviolet bands of OH fundamental data. J Quant Spectrosc Radiat Transf 2:97

13. Zhu X, Pu Y (2010) A simple collisional-radiative model for low temperature argon discharges with pressure ranging from $1 \mathrm{~Pa}$ to atmospheric pressure: kinetics of Paschen $1 \mathrm{~s}$ and $2 \mathrm{p}$ levels. J Phys D Appl Phys 43:17

14. Cho GS, Choi EH, Uhm HS (2011) Plasma wave propagation with a plasma density gradient. Phys Plasmas 18:034504

15. Cho GS, Choi EH, Uhm HS (2013) Plasma bullet as a plasma diffusion wave-packet in plasma jets. IEEE Trans Plasma Sci 41:1635

16. Lu X, Leipold F, Laroussi M (2003) Optical and electrical diagnostics of a non-equilibrium air plasma. J Phys D Appl Phys 36:2662

17. Ioana I, Popescu F (2010) Methods for online monitoring of air pollution concentration, in air quality. InTech, p 103

18. Benstaali B, Boubert P, Cheron BG, Addou A, Brisset JL (2002) Density and rotational temperature measurements of the $\mathrm{OH}$ and $\mathrm{NO}$ radicals produced by a gliding arc in humid air. Plasma Chem Plasma Process 22:553

19. Kwon BG, Yoon J (2009) Superoxide anion radical: principle and application. J Korean Ind Eng Chem 20:593

20. Holt RB, Mclane CK, Oldenberg O (1948) Ultraviolet absorption spectrum of hydrogen peroxide. J Chem Phys 16:225

21. Srivastava N, Wang C (2011) Effects of water addition on $\mathrm{OH}$ radical generation and plasma properties in an atmospheric argon microwave plasma jet. J Appl Phys 110:053304

22. Baeva M, Rackow K, Becker MM, Ehlbeck J, Loffhagen D (2011) Characterization of atmospheric pressure microwave plasma in $\mathrm{N}_{2} / \mathrm{O}_{2} / \mathrm{H}_{2} \mathrm{O}$ gas mixtures. Topic C9, 30th ICPIG, Belfast, Northern Ireland U.K.

23. Sarani A, Nikiforov AY, Leys C (2010) Atmospheric pressure plasma jet in $\mathrm{Ar}$ and $\mathrm{Ar} / \mathrm{H}_{2} \mathrm{O}$ mixtures: optical emission spectroscopy and temperature measurements. Phys Plasmas 17:063504

24. Kanazawa S, Kawano H, Watanabe S, Furuki T, Akamine S, Ichiki R, Ohkubo T, Kocik M, Mizeraczyk J (2011) Observation of $\mathrm{OH}$ radicals produced by pulsed discharges on the surface of a liquid. Plasma Sources Sci Technol 20:034010

25. Pangomm K, Baik KY, Nam MK, Rhim H, Choi EH (2013) Preferential killing of human lung cancer cell lines with mitochondria dysfunction by nonthermal dielectric barrier discharge plasma. Cell Death Dis 4:e642 